\title{
EFECTOS DE UN ENTRENAMIENTO EN LENGUAJE POSITIVE EN UN GRUPO DE PADRES DE FAMILIA
}

\author{
EFFECTS OF A TRAINING IN POSITIVE LANGUAGE IN A GROUP OF FAMILY \\ PARENTS
}

\author{
RICARDO ARBULÚ COLONA ${ }^{1}$
}

Universidad Nacional Mayor de San Marcos, Facultad de Psicología, Escuela de Post Grado

\section{RESUMEN}

OBJETIVOS: Los objetivos planteados apuntaron a modificar el lenguaje parental dentro del continuo positivo-negativo; describir los cambios en la percepción que los padres tienen de sí mismos, con referencia al estilo para expresarse; describir los cambios en la percepción que los hijos tienen de sus padres, con referencia al estilo para expresarse y por último describir los cambios en lo que respecta a la terminología más usada por los padres, con referencia a la forma de comunicarse con los hijos. DISEÑO: De Grupo único con medición antes y después, realizado en familias correspondientes a los alumnos del ler año de secundaria del Colegio América del Callao. MATERIAL y MÉTODOS: la muestra se basó en el criterio de discriminación por participación, se invitó a las 120 familias de los cuales se inscribieron 70, luego de un sorteo se seleccionaron 8 familias por aula, haciendo un total de 24. Se utilizó la Escala de lenguaje Parental y además se diseñó el Programa Padres Positivos, con el propósito de optimizar la conducta verbal de los padres con relación a sus hijos. RESULTADOS: Se aprecia que hubo cambios significativos en 4 de los 20 ítems de la Escala de Lenguaje Parental. CONCLUSIONES: Luego de aplicar el Programa PPP, se aprecia cambios en la V. D. Pudiendo concluir en lo siguiente: el Programa Padres Positivos, modifica la conducta verbal de los padres. Como detalle de esta afirmación podemos agregar que: Tanto desde la perspectiva de los padres participantes, como desde la perspectiva de sus hijos, se dio un cambio en la conducta verbal parental, considerando los puntajes globales.

Palabras Claves: PPP (Programa para padres positivos), lenguaje parental positivo.

\begin{abstract}
OBJECTIVES: The raised objectives aimed to inside modify the parental language of the continuous positive-negative; to describe the changes in the perception that the parents have of themselves, by referring to the style to express itself; to describe the changes in the perception that the children have of their parents, by referring to the style to express itself and finally to describe the changes with regard to the terminology more used by the parents, in reference to the form to communicate with the children. DESIGN: Of unique Group with measurement before and later, made in families corresponding to the students of $1 \mathrm{er}$ year of secondary of the America School of the Callao. MATERIAL AND METHODS: The sample was based on the criterion of discrimination by participation, invited the 120 families of who 70 registered, after a drawing a total of 24 was selected to 8 families by classroom, doing. The Scale of Parental Language was used and in addition the Positive Parent program was designed, in order to optimize the verbal conduct of the parents in relation to its children. RESULTS: It is appraised that there were significant changes in 4 of the 20 items of the Scale of Parental Language. CONCLUSIONS: After
\end{abstract}

${ }^{1}$ Doctorando de la Unidad de Post-grado de Psicología de la UNMSM 
applying Program PPP, it is appraised changes in V.D. Being able to conclude in the following thing: the Positive Parent program, modifies the verbal conduct of the parents. As it details of this affirmation we can add that. As much from the perspective of the participant parents, like from the perspective of its children, a change in the parental verbal conduct occurred, considering the scores global.

Keywords: PPP (Positive Parents Program), positive parental language.

\section{INTRODUCCIÓN}

El investigador empieza preguntándose acerca del probable efecto en la vida emocional de los niños, que pudiera tener las expresiones lingüísticas de parte de los padres.

Consciente de que la educación de los padres en el Perú y el mundo aún sigue un proceso de consolidación, se pretende por medio de la investigación aportar mejores métodos, creándolos o descubriéndolos y simultáneamente contribuir a la investigación de carácter teórico.

El tema resulta importante en la misma medida en que valoramos la importancia de la familia y sobre la base de admitir que el modelo más significativo de carácter emocional para un niño, es la figura parental.

Se quiere estudiar los efectos que tendría un entrenamiento en el uso de frases, caracterizadas por un lenguaje positivo. El entrenamiento está constituido por un seminario taller que tiene la denominación de PPP (Programas Padres Positivos)

Se formula el problema del modo siguiente: «¿En qué medida el programa padres positivos (PPP) Modifica la conducta verbal de los padres?»

Al buscar los antecedentes, observamos que más son los libros dirigidos a los padres acerca de cómo educar a sus hijos, y pocos son los libros dirigidos a psicólogos acerca de cómo educar a los padres, (lo segundo es el tema de interés de la presente investigación)

Respecto a la Educación de Padres en el Mundo, el estudio de H. STERN. (1961) ${ }^{1}$ auspiciado por la UNESCO, puede ser considerado como el más completo hasta la fecha. Por medio de él nos ilustra acerca de las distintas maneras en que ha intentado educar, las contradicciones que se observan, el aporte de los organismos internacionales y la educación de padres potenciales. Respecto a la Educación de Padres en el Perú, básicamente tenemos que acudir a estudios pragmáticos no publicados, pero si referidos por los autores, mediante informes y conferencias (Arbulú, 1991) ${ }^{2}$ hizo la experiencia en padres de niños pequeños (Kinder); Mayo, $(1995)^{3}$, lo hizo con padres de niños especiales.

Stern hace referencia a diversos modos de educar a los padres, históricamente asocia la idea con métodos de crianza, brindados en hospitales para los cuidados de la salud. Luego las formas han evolucionado hasta la organización de Clubes de Padres. Hace falta incluir métodos en el sistema de la Educación Escolarizada así como plantear estrategias para la Educación de Padres tomando como eje los medios de comunicación social.

Tomando como referencia algunas bases teóricas para poder explicar la relación funcional entre el PPP (Programa Padres Positivos) y la conducta verbal de los padres se acude a determinados conceptos básicos acerca del lenguaje. Las primeras teorías se refieren al proceso que sigue el lenguaje desde que es aprendido en función de una comunidad lingüística hasta que es interiorizado como pensamiento individual, tal como lo plantean Skinner $(1978)^{4}$.

Otras teorías, como el Análisis Transaccional de Eric Berne(1966) ${ }^{5}$, se refieren a la calidad del lenguaje aprendido (positivo - negativo) y analiza los efectos que se derivan de la calidad del lenguaje utilizados por los padres.

Sobre esa base se hace la propuesta del lenguaje positivo como alternativa, para lo cual se 
establecen los diferentes indicadores que permiten que el lenguaje positivo sea mejor entendido y manejado operacionalmente.(Arbulú, $1977^{6}$ ). Para efectos de este estudio, se define el "lenguaje positivo» como aquella comunicación humana, que se caracteriza por favorecer la concordancia entre las personas que se comunican y en consecuencia producir bienestar. Los componentes del lenguaje positivo, constituyen factores tales como: Democracia Emocional, Firmeza, Afecto, Eficiencia y Realismo.

Los indicadores del lenguaje positivo, que se proponen, se muestran en función de un continuo que polariza lo positivo (lenguaje mejor entendido y manejado operacionalmente. (Arbulú, 1977) ${ }^{6}$.

Para efectos de este estudio, se define el "lenguaje positivo» como aquella comunicación humana, que se caracteriza por favorecer la concordancia entre las personas que se comunican y en consecuencia producir bienestar. Los componentes del lenguaje positivo, constituyen factores tales como: Democracia Emocional, Firmeza, Afecto, Eficiencia y Realismo. Los indicadores del lenguaje positivo, que se proponen, se muestran en función de un continuo que polariza lo positivo (lenguaje que hace sentir bien) vs lo negativo (lenguaje que hace sentir mal) Así por ejemplo: la calificación, hace sentir bien, ejemplo: la calificación, hace sentir bien, en cambio la descalificación, hace sentir mal.

En la presente investigación se construye una definición operacional para lenguaje positivo, definiéndolo como el conjunto de frases utilizadas por los padres que se caracterizan por su tendencia a incentivar factores como: Autoestima de sus hijos, Autonomía para razonar y Expresión de sentimientos

Por el contrario el lenguaje parental negativo, es definido operacional mente como el conjunto de frases utilizadas por los padres que se caracterizan por su tendencia a: Disminuir la Autoestima, Generar dependencia y Expresar calificativos en lugar de sentimientos. Sobre la base de lo anterior se puede entender mejor la definición del PROGRAMA PADRES POSITIVOS (PPP) de la siguiente manera: "Seminario taller estructurado con el auxilio de la tecnología educativa, dirigido a los padres, con el propósito de entrenarlos en lenguaje parental positivo.

\section{Materiales y método}

Tipo de investigación

La presente investigación es de nivel explicativo y de tipo pre experimental

Diseño de investigación

Diseño de grupo único con medición antes y después

Población y muestra

Población compuesta por 120 familias correspondientes a los alumnos del 1er año de secundaria del Colegio América del Callao.

La muestra se basó en el criterio de discriminación por participación, se invitó a las 120 familias de los cuales se inscribieron 70, luego de un sorteo se seleccionaron 8 familias por aula, haciendo un total de 24 .

Los sujetos muestrales presentaron similar status socioeconómico de clase media, secundaria completa con residencia de no menos de 10 año en la zona y declarados católicos. 
Identificación de variables

Variables Independientes

Como variable experimental se constituyo el PPP

Variables dependientes:

- Lenguaje parental

- Imagen parental asociada a la conducta verbal

- Terminología más usada

Variables (externas), (controladas)

Los padres participantes pertenecen al mismo colegio, mismo nivel socioeconómico, misma religión, y sus hijos están en el mismo grado (1er año de secundaria).

\section{Técnicas e instrumentos de recolección de datos}

\section{Escala de Lenguaje Parental}

Contiene 10 frases que representan el lenguaje positivo y 10 frases que representan el lenguaje negativo, las cuales deben ser escogidas en un continuo de respuestas tipo Líker.

Esta escala fue validada utilizando el Método del ITEM-TEST, siendo previamente sometida a una validación de contenidos mediante jueces.

a. Encuesta $\mathrm{N}^{\circ} 1$ acerca de la Imagen Paterna

La organización de esta encuesta se basa en un criterio teórico que deviene del AT. Distingue dos modelos que caracterizan la forma en que se expresan los padres:

Modelo a: Padre Critico (negativo) Modelo b: Padre Protector (positivo)

El contenido de cada ítem no ha sido escogido por el investigador, sino que corresponda un esquema pre establecido por los especialistas del AT, siguiendo las ideas planteadas por u creador E. Berne ${ }^{5}$.

b. Encuesta $\mathrm{N}^{\circ} 2$ acerca de la «Terminología más usada»

En esta encuesta se ha seleccionado un conjunto de frases que correspondan a la concepción teórica según el AT acerca de los mensajes parentales. Dichos mandatos (que toman la forma de mandatos, impulsores o permisores) constituyen frases clasificadas por la teoría en un rubro positivo (nutritivo) o en rubro negativo (crítico).

Las frases en consecuencia no son producto de la imaginación del investigador sino más bien, extraídas del contexto teórico del AT.

c. Encuesta $\mathrm{N}^{\circ} 3$ acerca del "Testimonio de los padres»

Son siete preguntas abiertas al término del programa con la finalidad de evaluarlo en función de la libre opinión de los padres para redactar las respuestas. Con la idea de organizarla las respuestas, se clasificaron en tres áreas (cognitiva, afectiva $\mathrm{y}$ conductual). 
Instrumento de Intervención

(Descripción sucinta del PPP)

\section{Programa Padres Positivos}

\section{$\underline{\text { Objetivo }}$}

Este Programa se diseña en el propósito de optimizar la conducta verbal de los padres con relación a sus hijos. Se propone que los padres identifiquen expresiones lingüísticas negativas con la intención de cambiarlas por frases positivas.

Metodología

Para lograr este objetivo se acudió a la modalidad del Taller, a la que se sumó la modalidad de la Jornada y la técnica de la «Actividad Compartida» con los hijos.

\section{Didáctica}

Básicamente, la didáctica fue centrada en el participante, programando su participación activa, en función de reactivos, haciendo una dinámica con matices lúdicos. Se empleó la retroalimentación venida de los hijos mediante testimonios vivenciales y se emplearon abundantes reforzadores para crear un clima grato y positivo a los padres participantes.

Se empleó las ayudas audiovisuales que brindó al colegio.

Temática Básica

En síntesis se desarrolló el tema del afecto teniendo como instrumento el lenguaje positivo. Se analizó las experiencias de Harlow $(1930)^{7}$ relativas a monos con carencia de contacto inter-individual, lo cual permitía apreciar el valor de la calidad de la relación padre-hijo y sus consecuencias.

Se proporcionó técnicas verbales orientadas a elevar la Autoestima tales como los elogios y las caricias psicológicas.

\section{RESULTADOS}

Los resultados se presentaran en la siguiente secuencia:

$1^{\circ} \quad$ Resultados Correspondientes a la escala de Lenguaje Parental

Como complemento se tocó el tema de «cómo ayudar a reforzar los hábitos de estudio». Asimismo, mediante un taller específico acerca de la «vivencia del barro» se trabajó la actitud emocional hacia los hijos.

Organización

Se estableció un planeamiento en función de guías didácticas, un calendario de actividades y un seguimiento posterior. Más detalles del Programa Padres Positivos (PPP) se explican en la sección de anexos.

\section{RESULTADOS GLOBALES}

Resumen: Existe diferencia significativa entre los puntajes antes y después lo cual se puede apreciar en la Tabla 1 correspondiente a los padres y en la Tabla 2 correspondiente a los hijos de los padres participantes.

Tabla 1.- Valores del test $t$ alcanzado por los padres participantes en la «Escala de Lenguaje Parental" antes y después del programa experimental.

\begin{tabular}{|c|c|c|c|c|c|c|c|}
\hline & $\mathrm{N}$ & $\mathrm{X}$ & $\mathrm{Ds}$ & $\mathrm{Ds}^{2}$ & Dif. & $\sigma_{\mathrm{D}}$ & $\mathrm{t}$ \\
\hline MO1 & 24 & 3.05 & 3.36 & 11.29 & \multirow{2}{*}{3.9} & 1.04 & $3.75^{*}$ \\
\hline MO2 & 24 & 34.4 & 3.81 & 14.52 & & & \\
\hline \multicolumn{8}{|c|}{$* p<.05$ t critico $=2.70$}
\end{tabular}


Tabla 2.- Valores del test "t» alcanzado en la «Escala de Lenguaje Parental», por los hijos de los participantes antes y después del programa experimental.

\begin{tabular}{|l|c|c|c|c|c|c|c|}
\hline & $\mathrm{N}$ & $\mathrm{X}$ & $\mathrm{Ds}$ & $\mathrm{Ds}^{2}$ & Dif. & $\sigma_{\mathrm{D}}$ & $\mathrm{t}$ \\
\cline { 1 - 5 } MO1 & 24 & 30.20 & 3.08 & 9.49 & 3.6 & \multirow{2}{*}{0.74} & 3.51 \\
\hline MO2 & 24 & 32.80 & 1.89 & 1.89 & & & \\
\hline \multicolumn{8}{|c|}{$* 5$ t crítico $=2.70$}
\end{tabular}

\section{RESULTADOS ESPECIFICOS}

Resumen: Los items que alcanzan puntajes significativos en los padres son 1, 3, 5 y 9 (Tabla 5) En los hijos 1, 3 y 11 (Tabla 6)

Tabla 3.- Grado de Significación de los items de la Escala de Lenguaje Parental. Muestra: Padres que participaron en el taller

FRASES USADAS DELANTE DEL HIJO

1."Aquí mando yo, los demás obedecen" $\mathrm{X} 2$ sig.

2. "Confío en que podrás superarte"

3. "iSiéntate y estudia!"

4. "Muy bien te felicito, ¡Bravo!"

5. "Eres un flojo, ocioso, haragán!"

6. "Yo sé que tu puedes"

7. "No sirves para nada"

0

8. "Adelante vamos, anímate"

0.25

9. "Nunca serás un buen estudiante"

$5.1 *$

10. "No te preocupes, cualquiera se equivoca"

0

11. "QQué bueno eres"”

12. "¿Qué necesitas, te traigo algo?"

1.3

13. " $¡ N a d a$ te sale bien"

14. “En que puedo ayudarte?”

15. “¿Y? .. Cuántos rojos esta vez?”

16. "Estoy orgulloso de ti"

17. "Te equivocaste iqué sonzo!"

18. "Me preocupo cuando no llegas"

19. "Sufro cada vez que tengo que ver tus notas"

20. "Te extraño mucho"

2.2

* Valor crítico de X2 al 0.05 con $1 \mathrm{gl}=3.84$

* Valor crítico de X2 al 0.05 con $1 \mathrm{gl}=3.84$ 
Tabla 4.- Grado de Significación de los items de la Escala de Lenguaje Parental. Muestra: Hijos de los Padres que participaron en el taller

FRASES USADAS DELANTE DEL HIJO

1."Aquí mando yo, los demás obedecen"

2. "Confío en que podrás superarte"

3. "iSiéntate y estudia!"

$\begin{array}{ll}\mathrm{X} 2 & \text { sig. } \\ 4 . & * \\ 0 & \\ 4.9 & *\end{array}$

4. "Muy bien te felicito, ¡Bravo!"

$4.9 *$

5. "Eres un flojo, ocioso, haragán!"

6. "Yo sé que tu puedes"

0

7. "No sirves para nada"

8. "Adelante vamos, anímate"

9. "Nunca serás un buen estudiante"

10. "No te preocupes, cualquiera se equivoca"

11. "¡Qué bueno eres"”

0.50

12. “Qué necesitas, te traigo algo?"

13 "¡Nada te sale bien"

14. “¿En qué puedo ayudarte?"

15. “YY? Cuántos rojos esta vez?”

16. "Estoy orgulloso de ti"

17. "Te equivocaste ¡qué sonzo!"

18. "Me preocupo cuando no llegas"

0.8

19. "Sufro cada vez que tengo que ver tus notas"

0

20. "Te extraño mucho"

* Valor crítico de X2 al 0.05 con 1 g.l $=3.84$

\section{RESULTADOS EN FUNCIÓN AL GÉNERO}

Resumen: Existen diferencias significativas entre los momentos antes y después, tanto en las respuestas de los padres (Tabla 5) como la de los hijos (Tabla 6) percibidas en todos los casos. Aparentemente los hijos manifiestan que los papás cambiaron más. (Tabla 6).

Tabla 5.- Diferencia de medias aritméticas en la «Escala de Lenguaje Parental» percibida por los padres participantes, según el género.

\begin{tabular}{|c|c|c|c|c|c|c|c|}
\hline & $\mathrm{N}$ & $X$ & Ds & Ds2 & Dif. & $\sigma_{\mathrm{D}}$ & $\mathrm{t}$ \\
\hline Padres & & & & & \multirow{3}{*}{3.52} & \multirow{3}{*}{1.16} & \multirow{3}{*}{$3.03 *$} \\
\hline MO1 & 8 & 30.4 & 32.54 & 6.45 & & & \\
\hline MO2 & 8 & 33.92 & 2.09 & 4.37 & & & \\
\hline \multicolumn{5}{|l|}{ Madres } & \multirow{3}{*}{4.7} & \multirow{3}{*}{1.20} & \multirow{3}{*}{$3.31 * *$} \\
\hline MO1 & 16 & 30.5 & 3.04 & 10.33 & & & \\
\hline MO2 & 16 & 35.2 & 3.6 & 12.96 & & & \\
\hline
\end{tabular}

*valor critico significativo $\mathrm{p}<.05, \mathrm{gl}=14, \mathrm{t}$ critico $=2.977$

$* *$ valor critico significativo $\mathrm{p}<.05, \mathrm{gl}=30, \mathrm{t}$ critico $=2.750$ 
Tabla 6. Diferencia de medias aritméticas en la «Escala de Lenguaje Parental» percibida por los hijos de los padres participantes, según el género.

\begin{tabular}{|c|c|c|c|c|c|c|c|}
\hline & $\mathrm{N}$ & $X$ & Ds & Ds2 & Dif. & $\sigma_{\mathrm{D}}$ & $\mathrm{t}$ \\
\hline Padres & & & & & \multirow{3}{*}{4.9} & \multirow{3}{*}{1.21} & \multirow{3}{*}{$4.04 *$} \\
\hline MO1 & 8 & 29.5 & 2.7 & 7.29 & & & \\
\hline MO2 & 8 & & & & & & \\
\hline Madres & & & & & \multirow{3}{*}{3.2} & \multirow{3}{*}{1.02} & \multirow{3}{*}{$3.14 * *$} \\
\hline MO1 & 16 & 31.2 & 2.9 & 8.41 & & & \\
\hline MO2 & 16 & 34.4 & 2.8 & 9.12 & & & \\
\hline
\end{tabular}

$2^{\circ}$ Resultados correspondientes a la Escala $N^{\circ} 1$ acerca de, la $<<$ Imagen Paterna"

\section{RESULTADOS GLOBALES}

Resumen: Existe diferencia entre antes y después tanto en padres como en los hijos, sin embargo los padres perciben que el cambio es mayor.

Tabla. 7.- Diferencia de medias aritméticas en la encuesta acerca de la "imagen Parental» percibida por los padres participantes y por sus hijos.

\begin{tabular}{|c|c|c|c|c|c|c|c|}
\hline & $\mathrm{N}$ & $X$ & Ds & Ds2 & Dif. & $\sigma \mathrm{D}$ & $\mathrm{t}$ \\
\hline Padres & & & & & \multirow{3}{*}{4} & \multirow{3}{*}{0.90} & \multirow{3}{*}{4.44} \\
\hline MO1 & 24 & 4 & 2 & 4 & & & \\
\hline $\mathrm{MO} 2$ & 24 & 8 & & & & & \\
\hline Madres & & & & & \multirow{3}{*}{2} & \multirow{3}{*}{0.73} & \multirow{3}{*}{2.74} \\
\hline MO1 & 24 & 4 & 2 & 4 & & & \\
\hline MO2 & 24 & 6 & 3 & 9 & & & \\
\hline
\end{tabular}

\section{RESULTADOS ESPECIFICOS}

Resumen: Cambio en el ítem $\mathrm{N}^{\circ} 2$ referido al tono de voz. Tal resultado es coincidente entre los padres (Tabla 8) y los hijos (Tabla 9) 
Tabla 8.- Grado de Significación de los items de la Escala de Imagen Parental Muestra: Padres que participaron en el taller

\begin{tabular}{|c|c|c|c|c|}
\hline & \multirow{2}{*}{\multicolumn{2}{|c|}{ MODELO PARENTAL }} & \multirow{3}{*}{$\mathrm{X} 2$} & \multirow{4}{*}{ Sig } \\
\hline & & & & \\
\hline & MODELO A & MODELO B & & \\
\hline 1. Palabras que usa & $\begin{array}{c}\text { TU DEBERIAS } \\
\text { TIENES QUE } \\
\text { NO SEAS RIDICULO }\end{array}$ & $\begin{array}{l}\text { TU PUEDES } \\
\text { EREZ CAPAZ } \\
\text { TE APRECIO }\end{array}$ & 1.2 & \\
\hline 2.Tono de voz & $\begin{array}{l}\text { CRITICO } \\
\text { CORTANTE } \\
\text { BURLON }\end{array}$ & $\begin{array}{l}\text { CORDIAL } \\
\text { CARIÑOSO } \\
\text { CALIDO }\end{array}$ & 4.1 & $*$ \\
\hline 3. Expresión facial & $\begin{array}{c}\text { MOLESTA } \\
\text { CEÑO FRUNCIDO }\end{array}$ & $\begin{array}{l}\text { CONTENTA } \\
\text { SONRIENTE }\end{array}$ & 0 & \\
\hline 4.Gestos & $\begin{array}{l}\text { DEDO ACUSADOR } \\
\text { BRAZOS CRUZADOS } \\
\text { PUÑOS EN CADERAS }\end{array}$ & $\begin{array}{c}\text { BRAZOS ABIERTOS } \\
\text { BRAZOS QUE } \\
\text { RODEAN }\end{array}$ & 1.2 & \\
\hline 5. Postura corporal & $\begin{array}{c}\text { TRONCO ERGUIDO } \\
\text { RIGIDO }\end{array}$ & $\begin{array}{c}\text { TRONCO } \\
\text { INCLINADO } \\
\text { HACIA OTROS }\end{array}$ & 0.75 & \\
\hline 6.Actitud general & $\begin{array}{c}\text { AUTORITARIO } \\
\text { REÑIDOR }\end{array}$ & $\begin{array}{c}\text { COMPRENSIVO } \\
\text { SOLICITO, ATENTO }\end{array}$ & 2.3 & \\
\hline
\end{tabular}

*Valor critico de X2 al 0.05 con 1 g.l=3.84 
Tabla 9.- Grado de Significación de los items de la Encuesta de Imagen Parental Muestra: Hijos de los padres que participaron en el taller

\begin{tabular}{|c|c|c|c|c|}
\hline & \multicolumn{2}{|c|}{ MODELO PARENTAL } & \multirow[t]{2}{*}{$\mathrm{X} 2$} & \multirow[t]{2}{*}{ Sig } \\
\hline & MODELO A & MODELO B & & \\
\hline 1. Palabras que usa & $\begin{array}{c}\text { TU DEBERIAS } \\
\text { TIENES QUE } \\
\text { NO SEAS RIDICULO }\end{array}$ & $\begin{array}{l}\text { TU PUEDES } \\
\text { EREZ CAPAZ } \\
\text { TE APRECIO }\end{array}$ & 2.2 & \\
\hline 2.Tono de voz & $\begin{array}{l}\text { CRITICO } \\
\text { CORTANTE } \\
\text { BURLON }\end{array}$ & $\begin{array}{l}\text { CORDIAL } \\
\text { CARIÑOSO } \\
\text { CALIDO }\end{array}$ & 3.88 & $*$ \\
\hline 3. Expresión facial & $\begin{array}{c}\text { MOLESTA } \\
\text { CEÑO FRUNCIDO }\end{array}$ & $\begin{array}{l}\text { CONTENTA } \\
\text { SONRIENTE }\end{array}$ & 0 & \\
\hline 4.Gestos & $\begin{array}{l}\text { DEDO ACUSADOR } \\
\text { BRAZOS CRUZADOS } \\
\text { PUÑOS EN CADERAS }\end{array}$ & $\begin{array}{c}\text { BRAZOS ABIERTOS } \\
\text { BRAZOS QUE RODEAN }\end{array}$ & 0.75 & \\
\hline 5. Postura corporal & $\begin{array}{l}\text { TRONCO ERGUIDO } \\
\text { RIGIDO }\end{array}$ & $\begin{array}{c}\text { TRONCO INCLINADO } \\
\text { HACIA OTROS }\end{array}$ & 3.1 & \\
\hline 6.Actitud general & $\begin{array}{l}\text { AUTORITARIO } \\
\text { REÑIDOR }\end{array}$ & $\begin{array}{c}\text { COMPRENSIVO } \\
\text { SOLICITO, ATENTO }\end{array}$ & 0.75 & \\
\hline
\end{tabular}

Valor critico de $X 2$ al 0.05 con 1 g.l=3.84

$3^{\circ}$ Resultados correspondientes a la encuesta $N^{\circ} 2$ acerca de, la «Terminología más usada» 


\section{RESULTADOS GLOBALES}

Resumen: Ambos grupos (Tabla 10) (Padres e Hijos observan los cambios, con relación al criterio Imagen Parental) los hijos más que los padres.

TABLA 10.- Diferencia de medias aritméticas en la encuesta acerca de la «Imagen Parental, percibida por los padres participantes y por sus hijos.

\begin{tabular}{|c|c|c|c|c|c|c|c|}
\hline & $\mathbf{N}$ & $\mathbf{X}$ & Ds & Ds2 & Dif. & $\sigma_{\mathrm{D}}$ & $\mathbf{t}$ \\
\hline Padres & & & & & \multirow{3}{*}{2.45} & \multirow{3}{*}{2.45} & \multirow{3}{*}{$4.15^{*}$} \\
\hline MO1 & 24 & 3.05 & 2.04 & 4.16 & & & \\
\hline MO2 & 24 & 5.5 & 2.09 & 4.36 & & & \\
\hline Madres & & & & & \multirow{3}{*}{4.0} & \multirow{3}{*}{0.67} & \multirow{3}{*}{$5.97 *$} \\
\hline MO1 & 24 & 2.5 & 2.04 & 4.16 & & & \\
\hline MO2 & 24 & 6.5 & 2.6 & 6.76 & & & \\
\hline
\end{tabular}

\section{RESULTADOS ESPECIFICOS}

Resumen: Los padres (Tabla 11) indican cambios en el ítem $\mathrm{N}^{\circ} 1$. Los hijos (Tabla 12) indican cambios en los ítem 1, 2 y 4, con relación a la Escala de Frases Usadas por los Padres. 


\section{TABLA. 11.- Grado de significación de los items de la encuesta referida al área de «Terminología parental más usada»}

\section{Muestra: Padres que participaron en el taller}

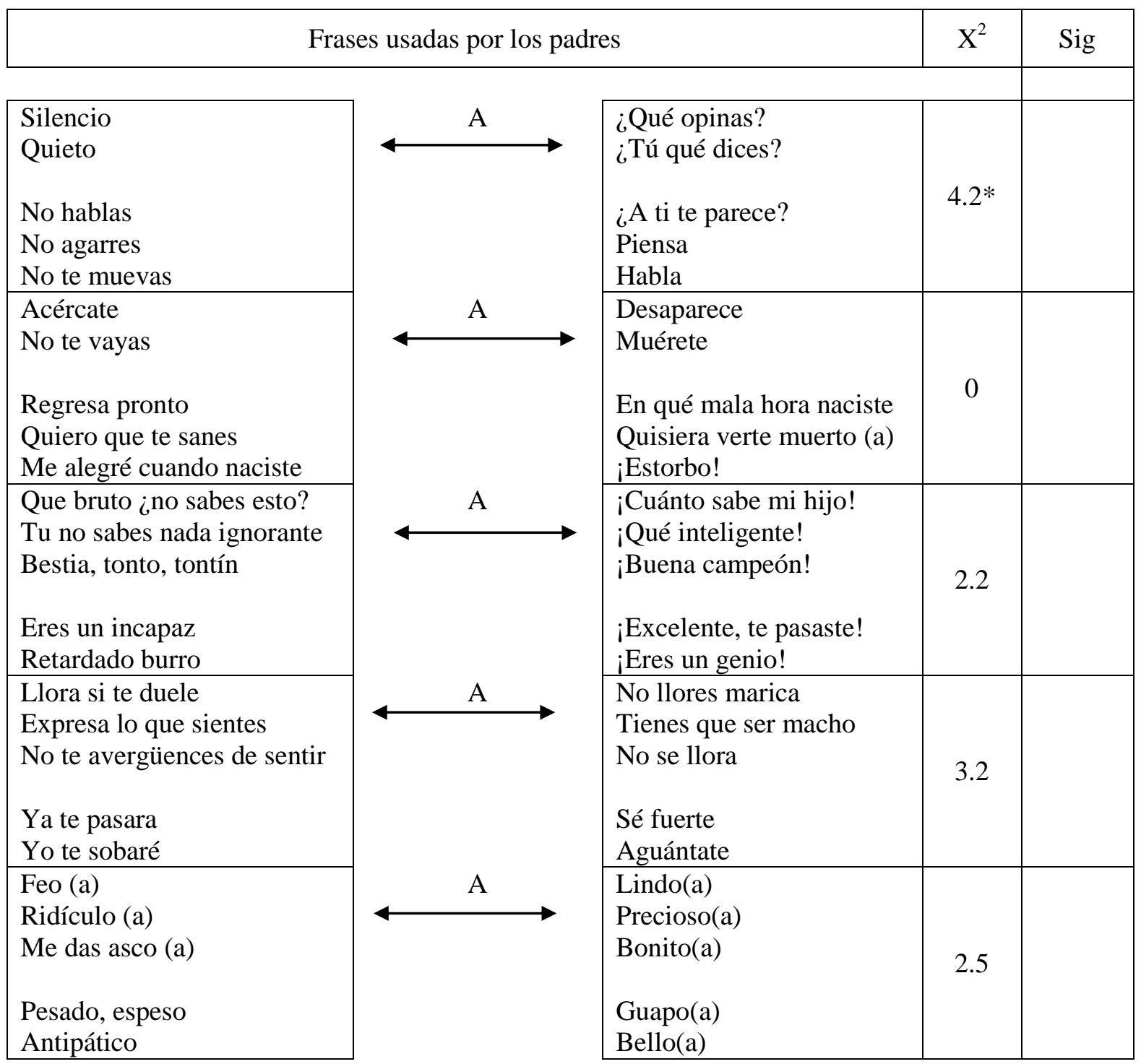

Valor critico de $X^{2}$ al 0.05 con 1 g.l=3.84 


\section{TABLA 12.- Grado de significación de los items de la encuesta referida al área de «Terminología parental más usada»}

\section{Muestra: Hijos de los padres que participaron en el taller}

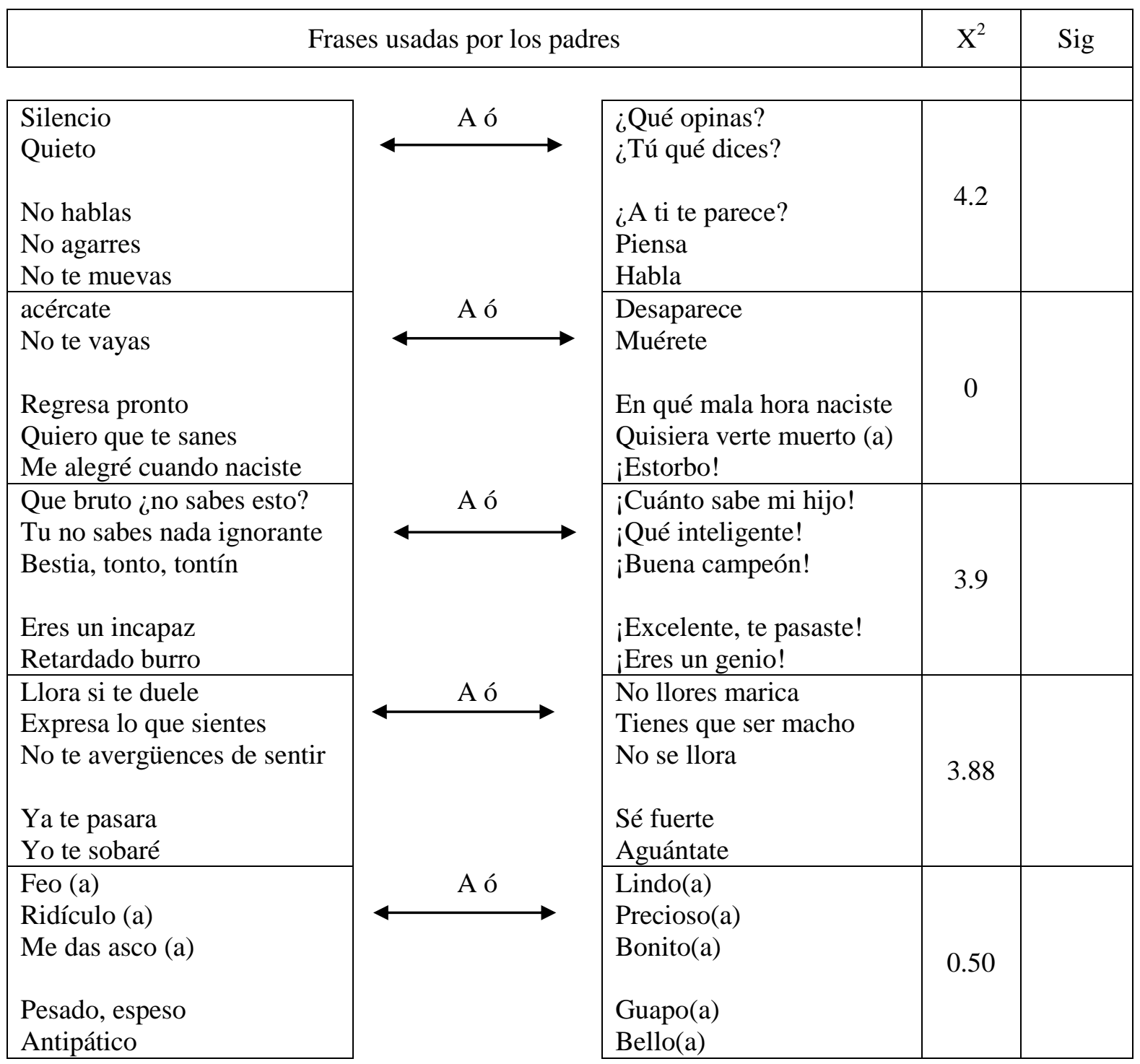

Valor critico de $X^{2}$ al 0.05

con 1 g.l $=3.84$

$4^{\circ}$ Resultados correspondientes a la encuesta $\mathrm{N}^{\circ} 3$ acerca de "Testimonio de los padres».

Se presentan en 7 cuadros, en los que se clasifica las respuestas de los padres en tres áreas (cognitiva, afectiva y conductual, para cada una de las 7 preguntas. El detalle de este resultado, el cual es de corte cualitativo, se puede apreciar en el Anexo 1 


\section{DISCUSIÓN}

En esta investigación hemos tenido como consecuencia un cambio de la V. D. debido a la aplicación de la V. I.

* ¿Por qué ha ocurrido este cambio? Existe una razón de carácter didáctico, si tomamos en cuenta el enfoque relativo a la Tecnología Educativa. (Ortiz, 1992) ${ }^{8}$. Enseñar a los padres supone plantearse objetivos, estructura temática, evaluaciones, en suma, planificar. Pero además se requiere establecer una metodología de enseñanza, sobre la base de diversas técnicas implementadas en el proceso de enseñanza aprendizaje. Debido a ello se interpreta que la razón del cambio en el caso de esta investigación se debe principalmente a la forma como está organizado el PPP (Programa Padres Positivos) el cual está compuesto por las siguientes características:

1. Participación activa de los padres en función de la dinámica de grupos.

2. Formación de grupos de trabajos, atendidos en diferentes días en forma independiente.

3. Empleo de reforzado res materiales (premios), sociales (reconocimiento), psicológicos (caricias verbales frecuentes)

4. Papel de facilitador del conductor.

Distinto al rol tradicional de expositor.

5. El tema seleccionado: (cómo lograr una comunicación positiva con los hijos) fue de interés para todos los padres

6. Proposición de un objetivo tangible: Uso de frases (que utilizamos en cantidad y en calidad)

7. Retroalimentación constante.

Comentario de los logros alcanzados en cada sesión luego de haber aplicado en casa las frases aprendidas.

Hasta aquí se puede afirmar que es factible que los padres modifiquen sus expresiones verbales negativas gracias a la influencia de un entrenamiento en lenguaje parental positivo.

En el supuesto que el contenido del PPP varíe su contenido, entonces se podrá variar las hipótesis, puesto que las variables dependientes serían diferentes. Posiblemente se quiera investigar el cambio que se daría en opciones múltiples (Autoestima, rendimiento académico, habilidades para el aprendizaje, relaciones interpersonales, inteligencia emocional, etc.) (Habría más tesis de corte experimental, ya que la mayoría por ahora son de carácter descriptivo)

*Por otro lado la investigación ha permitido confirmar ciertos conceptos teóricos tales como: Programación y Reprogramación. Así tenemos que un impulso como «apúrate» considerado dentro de la programación psicológica de un niño, se puede reprogramar empleando un permisor por medio de las frases como: «Tranquilo» «tómate tu tiempo» «piensa antes de hacer o contestan». (Kertész, 1993) ${ }^{9}$

El supuesto teórico, que remarca la importancia del afecto (Harlow, 1930) ${ }^{7}$, coincide con los planteamientos del AT, acerca de la «caricia positiva» (Villegas, 1983) ${ }^{10}$ En consecuencia se espera niños que sean más saludables socialmente, que se puedan relacionar mejor con sus pares. Por medio del estudio hemos verificado la importancia de la presencia de una comunidad lingüística que permite el cambio de la- conducta verbal, considerada como una operante conforme la teoría de Skinner ${ }^{4} \cdot$

* Los hallazgos pueden tener trascendencia si se entiende su aplicación precisamente a la comunidad que rodea al niño:

Instituciones y medios de comunicación social, más aún si del PPP va acompañado de otras 
programas preventivos dirigidos al niño en paralelo con los padres. La terminología que se emplea es sencilla y de fácil aplicación popular, por ejemplo para indicar el objetivo de formar un niño autónomo, productivo y feliz, los padres simplemente mencionan que el objetivo es formar un «hijo príncipe» lo cual resulta mucho más didáctico, que usar términos académicos.

* Si se quisiera realizar una réplica de esta investigación a futuro, vale la pena considerar la evaluación de otras variables por ejemplo rendimiento académico, autoestima, salud personal, ansiedad, manejo del estrés, que son consideraciones que sugiere Gottman $(1993)^{11}$ Habiendo hecho estudios acerca de la inteligencia emocional, entrenando a los padres para que a su vez entrenen a sus hijos, observó que tales entrenamientos tenían como efecto, mejoras en su salud, en sus calificaciones y en su vida social. El mérito de Gottman $^{11}$ y asociados es que estos estudios han sido hechos a largo plazo, y con una metodología que supone una gran inversión de recursos.

Cabe decir que para el caso de tomar en cuenta nuevas variables tendremos que tener los instrumentos de medición para poder verificar los cambios.

*En cuanto a los aportes que proporciona la investigación se puede afirmar que la comunidad profesional se beneficia con una prueba para el diagnóstico del Lenguaje Parental y anexos como son las encuestas acerca de la imagen paterna asociada a la conducta verbal y la terminología más usada por los padres.

Asimismo, constituye un aporte el instrumento responsable del cambio: el PPP cuyo diseño didáctico apunta a superar los métodos que hasta hoy venimos realizando con éxito relativo en el medio escolar regular. Aún cuando la «Escala de Lenguaje Parental «y el «PPP» son perfectibles, lo cierto es que desde ya se sienta una base para un trabajo psicológico, profesional y científico, de modo que se siga perfeccionando en el camino la educación para padres.

La idea ya está sembrada.

\section{CONCLUSIONES}

1. El PROGRAMA PADRES POSITIVOS modifica la conducta verbal de los padres. Como detalle de esta afirmación podemos agregar que:

a) Tanto desde la perspectiva de los padres participantes, como de la perspectiva de sus hijos, se dio un cambio en la conducta verbal considerando los puntajes globales.

b) En cuanto a los resultados específicos se aprecia que hubo cambios significativos en 4 de los 20 ítems de la Escala de Lenguaje Parental. Padres e hijos

2. Los hijos modifican la percepción que tienen de sus padres con relación a su conducta verbal, luego de que éstos participaran en el PPP.

3. Los padres cambiaron positivamente la «terminología más usada» el lenguaje hacia sus hijos como efecto del PPP. 


\section{REFERENCIAS}

1. Stern, H. La Educación de Padres. Buenos Aires: Kapelusz, 1961

2. Arbulú, R. Educación de Padres. Lima:

Biblioteca de la Maestría en Psicología. San Marcos (Monografía), 1992

3. Arbulu, R. Actitudes de los padres que tienen niños especiales. San Marcos (Monografía), 1992

4. Skinner, B. F. Conducta Verbal. México: Trillas, 1978

5. Berne, E. Los juegos en que participamos. Madrid: Javier Vergara Editor. 1966

6. Arbulu, R. Ser Mejor, Guía de Autoconocimiento. Lima: Grano de Mostaza, 1993

7. Harlow, H. Total social isolation in monkeys. Proc. Natl. Acad. Sci. USA 54, 90-97 1965.

8. Ortiz, Georgina. El significado de los colores. México, Trillas 1992

9. Kertesz, R. Introducción al Análisis Transaccional. Buenos Aires: Piados, 1973

10. Villegas, R. El tiempo y yo en un encuentro. México: Gala Editores, 1983

11. Gottman, J. The roles of conflict engagement, escalation, and avoidance in marital interaction: A longitudinal view of five types of couples. Journal of consulting and Clinical Psychology, 61, 6-15. 1993 